\title{
Computer aided optimisation of a constant shear-rate micro-channel contraction
}

\author{
Simon P. Higgins ${ }^{1} \quad$ Gregory J. Sheard ${ }^{2}$ \\ Andreas Fouras ${ }^{3} \quad$ Kerry Hourigan ${ }^{4}$
}

(Received 15 August 2008; revised 21 July 2009)

\begin{abstract}
A combination of spectral element computational fluid dynamics simulation and the Levenberg-Marquardt non-linear optimisation algorithm are used to optimise a micro-channel contraction. The Levenberg-Marquardt algorithm finds the optimum contraction profile which minimises changes in the shear rate near the channel centreline. The primary criterion for the design of the micro-channel contraction is a constant shear rate in the flow direction. This is motivated by the need to study living cells in an environment of uniform shear.
\end{abstract}

\section{Contents}

\section{Introduction}

C1005

http://anziamj.austms.org.au/ojs/index.php/ANZIAMJ/article/view/1462 gives this article, (c) Austral. Mathematical Soc. 2009. Published July 29, 2009. ISSN 1446-8735. (Print two pages per sheet of paper.) 


\section{Introduction}

Investigating the elasticity and rigidity of living cells answers many questions, including whether the cell is healthy or infected with a virus or pathogen [1]. Determining physical properties of cells such as elasticity and membrane toughness is non-trivial [2]. Cells are small, ranging from $3 \mu \mathrm{m}$ to $15 \mu \mathrm{m}$, and do not live long outside the host if not treated [3]. In the case of whole blood, cells need to be kept warm and in a nutritious environment whereas other elements of the blood will clot without the addition of anti-clotting agents. Therefore, there is a need to quickly interrogate cells for their rheological properties. In previous studies, individual cells were interrogated to characterise their mechanical properties [4]. However, current methods have changed little over the past 28 years. Typically a cell is held under a microscope, and a vacuum applied through a pipette to suck the cell up the pipette [5]. The further up the pipette the cell stretches, the higher its elasticity. This is a time consuming, manual process. Another method involves a solution with a high concentration of red blood cells (RBC) and a conventional rheostat [6]. Determining the viscosity of the solution allows investigators to deduce some mechanical properties of the RBC. The method that we propose involves passing the cells through a flow that exerts known forces on the cell. This process would then be captured via a digital camera with the cell deformation recorded and analysed via automated software.

We propose to photograph the cell membrane deformation due to the forces 
applied through a fluid flow. One such flow that is able to do this is a shearing flow within a channel [7]. The cell would be stretched by the drag of the slow flow compared to the force of the faster flow [8]. This shear gradient occurs when the flow interacts with a wall. For the forces to remain consistent on the cell as it flows downstream, the channel walls need to converge. This contraction yields a continuous rate of shear and the contraction boundaries define the shear rate. A simple flow to investigate this technique for cell interrogation, is a contraction with a constant rate of shear. This work provides a method to determine a boundary that produces the desired flow conditions.

The purpose of this investigation is to produce a flow with a known constant shear rate near the centreline. From symmetry the shear rate on the centreline will be zero. The form of shear that is being evaluated over the 2D channel is taken as the leading eigenvalue of Cauchy's strain tensor

$$
\dot{\varepsilon}=\frac{1}{2}\left(\frac{\partial u_{i}}{\partial y_{j}}+\frac{\partial v_{j}}{\partial x_{i}}\right),
$$

where $u$ and $v$ are the $x$ and $y$ components of the velocity field, respectively. The shear stress that is experienced by the cells in a Newtonian fluid is proportional to the viscosity of the working fluid and $\dot{\varepsilon}$. We investigate the method of producing this flow. We use an iterative process of solving for the flow and shear using a computation fluids dynamics (CFD) solver, and then use these data to assist in redefining the boundaries to converge on the desired result.

\section{Method}

The following section is a functional description of our computational code for solving this problem. Figure 1 illustrates the algorithm used to optimise the micro-channel contraction. Initial conditions including a starting mesh and required flow conditions are implemented before running through iterations 


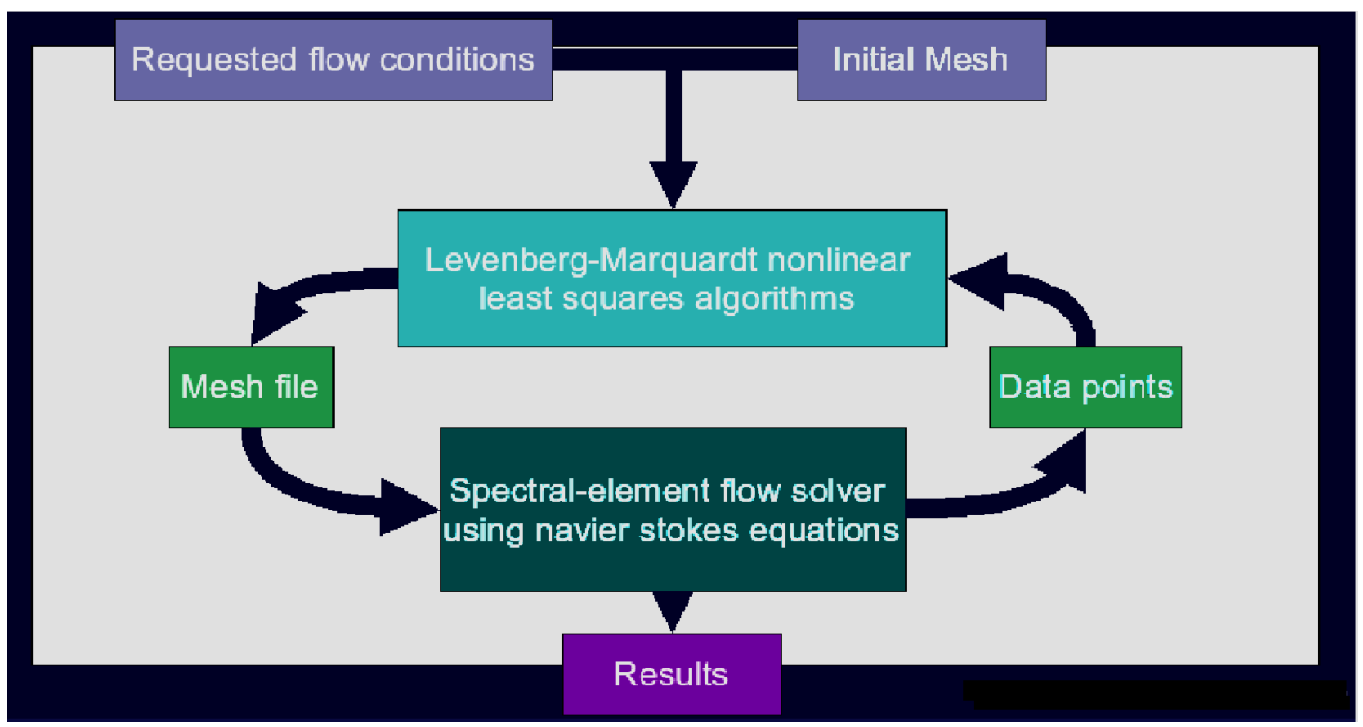

FIGURE 1: Illustration of the algorithm used to optimise the micro-channel contraction; starting with the initial mesh and required flow condition, running through iterations of flow solving and geometry optimisation, to produce the final result. 


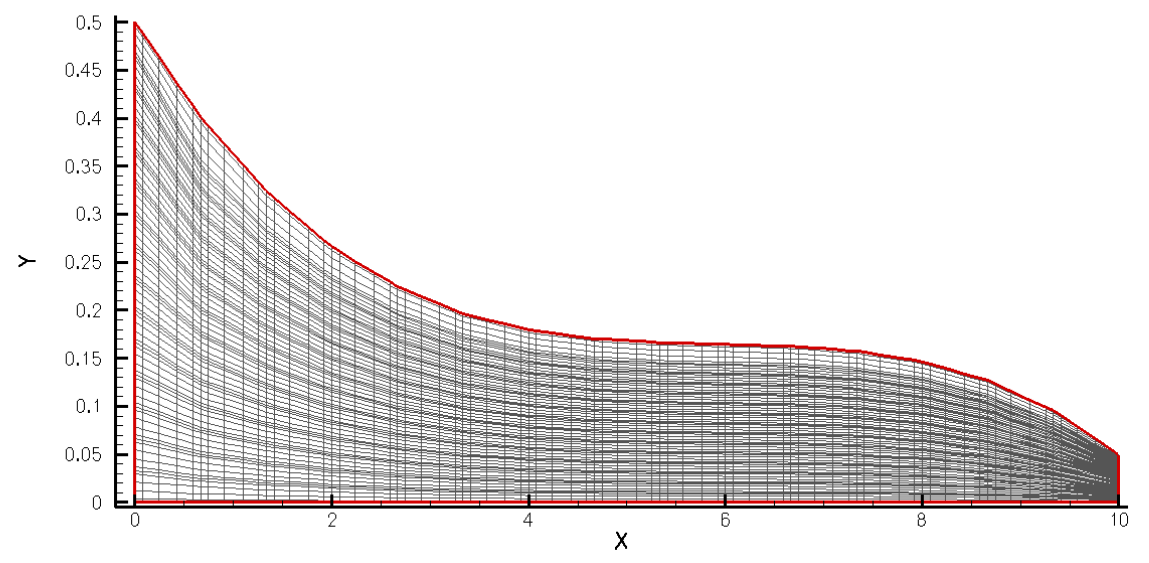

FiguRE 2: Illustration of the spectral element mesh used by the flow solver to compute shear rates. The top boundary profile is defined by the polynomial function in equation (2).

of flow solving and geometry optimisation, concluding with the final result. This code is a compilation of three major sections. The three sections are layered with the primary backbone of code holding the Levenberg-Marquardt (LM) solver, which in turn calls the CFD simulator that calculates the flow field. The starting input is a meshed geometry and the desired shear rates for the centreline. The flow field is then solved and the shear rates at discrete points (equivalent to half the radius of a $\mathrm{RBC}$ ) are evaluated before being passed into the LM solver. The LM solver then alters the geometry on the contraction and passes it to the CFD solver. This process is repeated until any of the following end conditions are met: the minimum error condition has been reached; the difference between changed geometries is below some threshold; or the maximum number of iterations has been reached.

The mesh manipulation routine has been written in the C programming language. Figure 2 shows the top boundary has a non-slip boundary condition 
applied, and this boundary is manipulated to change the contraction geometry. The boundary condition for the entrance on the left is a velocity profile in the $\mathrm{x}$-direction. The boundary on the right is the exit boundary and has a reference pressure boundary condition imposed on it. The bottom boundary along the $x$-axis has a symmetry boundary condition applied to it. The boundary that is being manipulated is defined by the third order polynomial

$$
y=a x^{3}+b x^{2}+c x+d,
$$

where $x$ and $y$ are the Cartesian coordinates with the origin, $(0,0)$, at the bottom left of the mesh in Figure 2. The function in equation (2) passes through four defining points, and two fixed points, one at the entrance and the other at the exit. The other two points are located at $x=2$ and at $x=8$. They are manipulated in their $y$-axis positioning to redefine the boundary geometry. The coordinates of the four points are used to calculate the constants $\mathbf{a}, \boldsymbol{b}, \boldsymbol{c}$, and $\mathbf{d}$ in equation (2). Removing the constraints on the fixed points leads to unrealistic channel geometries. This will be rectified in future development with elastic constraints. While the code is running, the boundary geometry is altered and remeshed in each iteration to ensure a suitable finite element model is defined for the flow solver. Figure 2 shows the smooth progression of element from the wall boundary to the centerline. The Reynolds number (Re) for this work is

$$
\mathrm{Re}=\frac{\mathrm{VD}}{v}=0.061,
$$

where $\mathrm{V}$ is the mean inlet velocity in $\mathrm{x}$-axis direction $\left(1.0 \times 10^{-3} \mathrm{~m} / \mathrm{s}\right)$, $\mathrm{D}$ is the height of the inlet of the channel $\left(0.2 \times 10^{-3} \mathrm{~m}\right)$, and $v$ is the kinematic viscosity of the fluid, $\left(3.3 \times 10^{-6} \mathrm{~m}^{2} / \mathrm{s}\right)$. Due to the fact that the Reynolds number for this flow is less than one, the solution is a creeping flow. This means the solver will reliably evolve the flow to time independent state. The steady state is defined to be when the change in velocity between time successive steps falls below $10^{-8}$. The code uses the centreline boundary as a plane of symmetry. This reduces computation time. The flow solver is a CFD 
package, developed in-house and described and validated by Sheard et al. [9], that solves the time dependent incompressible Navier-Stokes equations in either two or three dimensions. This package uses a spectral element method to discretize the Navier-Stokes equations in space, and employs a third order accurate, backwards multistep, method to evolve the solutions in time [10, 11]. For all simulations in this study, the fluid was modeled as a Newtonian fluid.

The Levenberg-Marquardt method works very well in practice and has become the standard of nonlinear least squares routines [12, p.683]. The algorithm finds the local minimum of a function that is expressed as the sum of squares of nonlinear functions, through an iterative process. This is demonstrated in the equation,

$$
S(\beta)=\sum_{i=1}^{m}\left[y_{i}-f\left(x_{i}, \beta\right)\right]^{2},
$$

where parameters $\beta$ of the model curve $f\left(x_{i}, \beta\right)$ are replaced at each iteration with $\beta+\delta$ until $S(\beta)$ is minimised, and $\delta$ is the parameter for each iteration. Differentiating equation (4) results in

$$
\left(J^{\mathrm{T}} \mathrm{J}\right) \delta=\mathrm{J}^{\mathrm{T}}[\mathrm{y}-\mathrm{f}(\beta)],
$$

using the first order approximation of $f\left(x_{i}, \beta+\delta\right)$ and setting the result to zero, since the gradient of the minimum point is zero by definition. In equation (5), $J$ is the Jacobian matrix whose ith row equals the gradient of $f$ with respect to $\beta$, and $f$ and $y$ are the vectors with ith component $f\left(x_{i}, \beta\right)$ and $y_{i}$ respectively. $J^{\top}$ is the transpose of $J$. This is a set of linear equations to be solved for $\delta$. The full Levenberg-Marquardt algorithm, as defined by

$$
\left[\mathrm{J}^{\top} \mathrm{J}+\lambda_{\text {diag }}\left(\mathrm{J}^{\top} \mathrm{J}\right)\right] \delta=\mathrm{J}^{\top}[\mathrm{y}-\mathrm{f}(\beta)],
$$

is adaptive as damping is controlled with $\lambda$. 


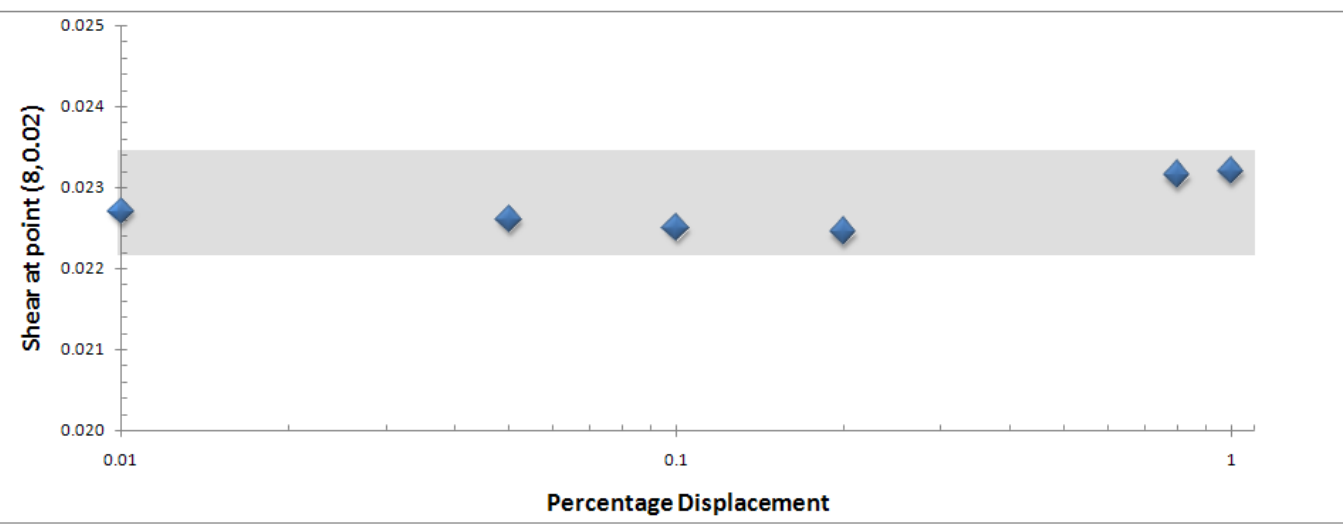

Figure 3: A plot of shear rates at location $(8,0.02)$ on the model against percentage displacements indicating the disturbance in the solution with regard to the change in sampling position. These data points have a standard deviation calculated to be $3.13 \times 10^{-5}$. The band highlights their position.

\section{Results}

Evaluation of the code has been conducted by looking at several variables to determine the stability of the code and to evaluate the degree of robustness. The variables evaluated included the number of sample points acquired, the position that the sample points were taken at, the sampling locations, the initial mesh arrangement and geometry. The number of samples used in the code was adjusted from 9 to 38. This allowed the determination of the error fluctuation as a function of the number of samples used. As the data points were being sampled from a spectral element mesh, this could lead to the position of the samples influencing the solution. This was found not to be the case. The initial mesh was altered and though this did have some effect on the convergence time there was no notable effect on the end result.

Figure 3 illustrates the deviation of the converged solutions. These data points have a standard deviation of $3.13 \times 10^{-5}$. 


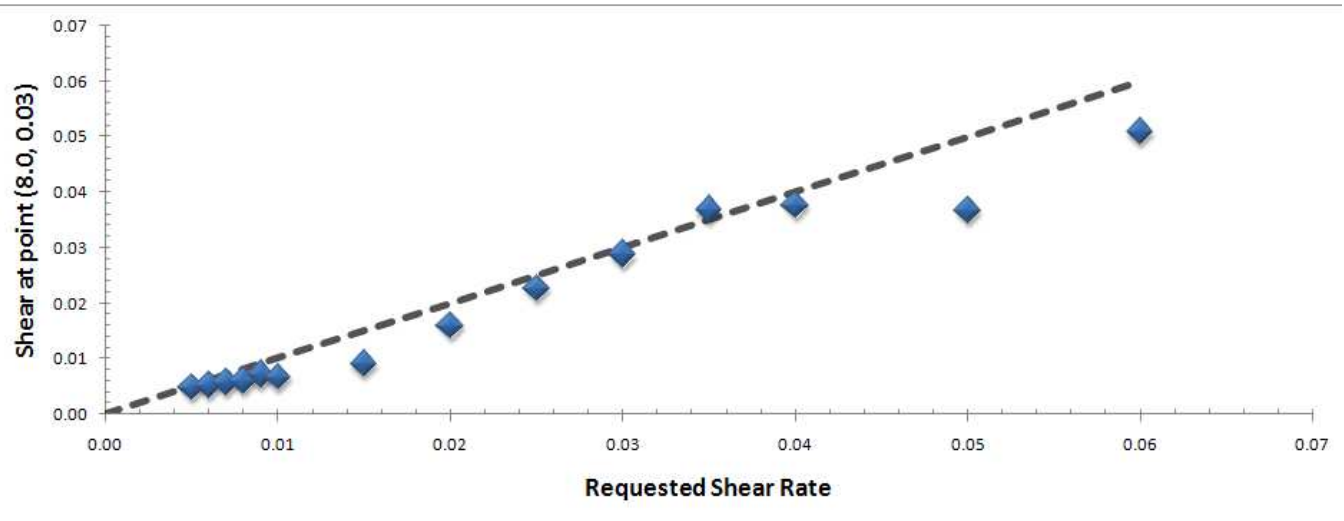

Figure 4: A plot of final computed shear rate against the desired shear rate. Shear rates below 0.005 and above 0.035 could not be attained as the channel inlet and outlet are fixed.

The solutions were evaluated by the extent to which the final solution minimised the error. Figure 4 illustrates the relationship between the computed and desired shear rates. A shear rate below 0.005 is not feasible as the outlet of the channel is fixed in this section and can not open out to allow lower shear rates. This graph also indicates the highest achievable shear with this fixed outlet, as the data asymptotes to 0.035 .

Figure 5 is a plot using a colour map to indicate the strain in the microchannel contraction after optimisation. Note that the channel is longer than displayed here, different scales are used on the horizontal and vertical axes. Figure 6 depicts a set of images of sequential iterations showing the code changing the geometry to meet the required flow conditions. For particular input conditions, this occurred over just six iterations, demonstrating the power of the LM solver. 


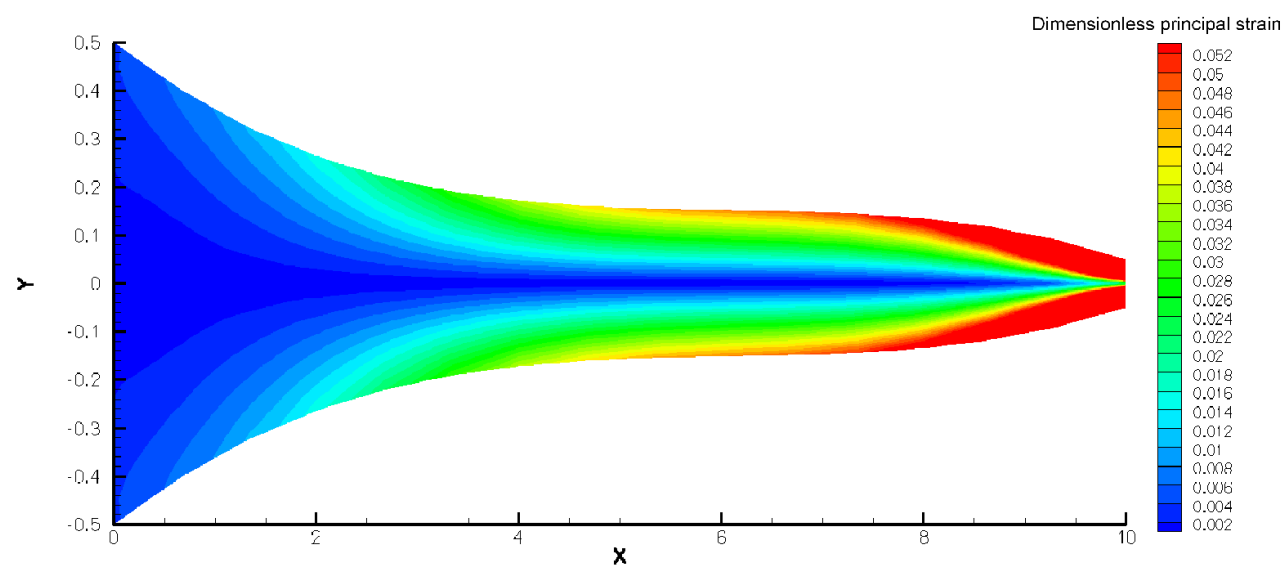

Figure 5: A Plot using a colour map to indicate the strain in the microchannel contraction after optimisation. Note that $x$ and $y$-axes carry different scales for convenience.

\section{Conclusion}

This study has shown the optimisation of parameters required for a third order polynomial boundary function to produce a constant shear rate on the centreline. This optimisation was achieved over just six iterations for the particular set of input conditions used, showing the power of the LM solver. The code and method both illustrate the robustness through convergence with little sensitivity to the number of samples or sample position. Only a small number of samples were needed to minimise the difference between resulting and desired shear rate. The robust nature of the code with respect to convergence lends confidence to ongoing work whereby the geometry is described by more complicated function such as cubic splines. This has contributed to improved micro-channel design where the specific flow requirement are designed into the system. 

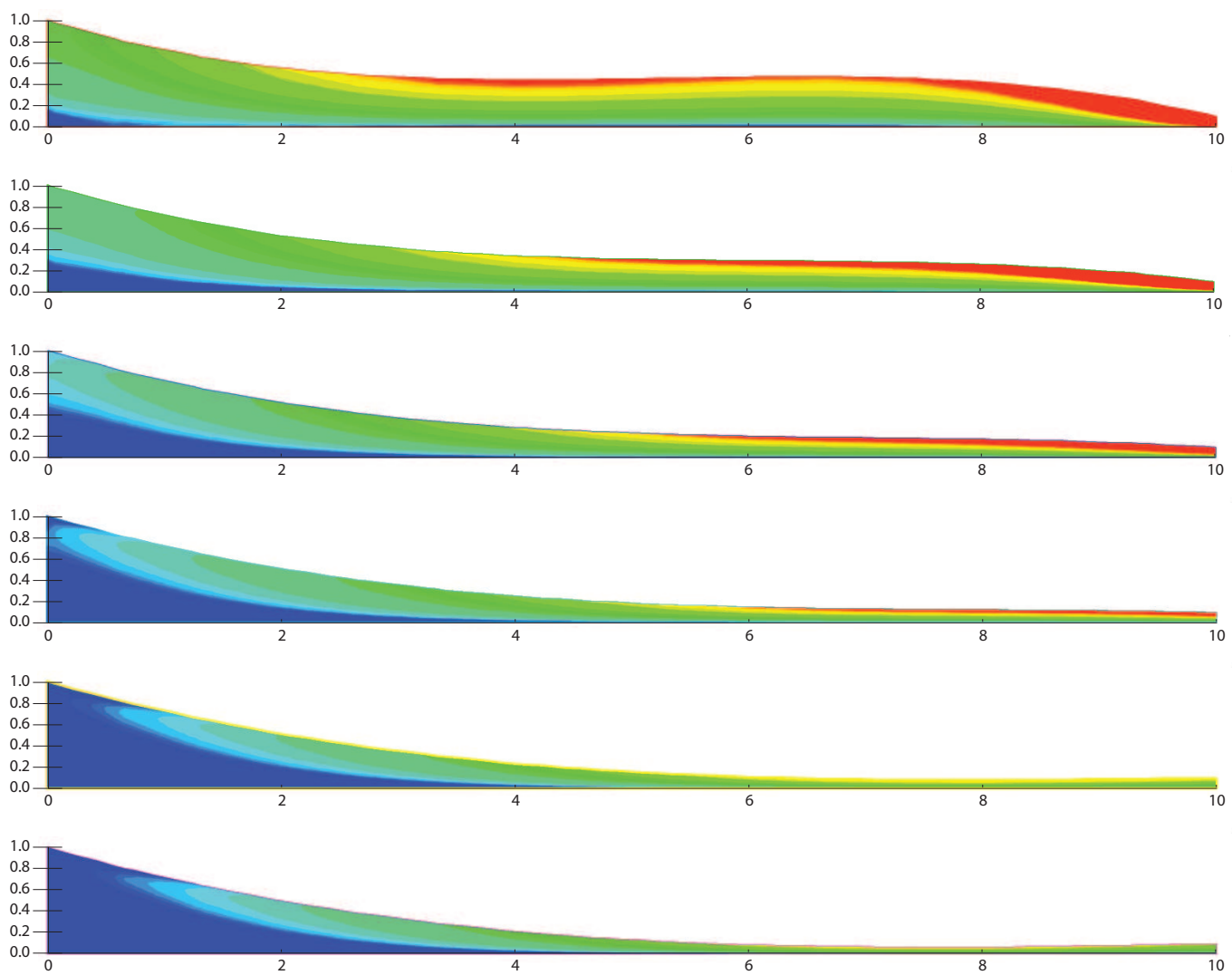

Figure 6: This set of images are sequential iteration steps of the code changing the geometry to meet the required flow conditions. This occurred over only six iteration shows the power of using the LM solver. 


\section{References}

[1] Popescu, G., Park, Y., Choi, W., Dasari, R. R., Feld, M. S., Badizadegan, K., Imaging red blood cell dynamics by quantitative phase microscopy, Blood Cells, Molecules, and Diseases, 41, 2008, 10-16. doi:10.1016/j.bcmd.2008.01.010 C1005

[2] Lee, S. S., Yim, Y., Kim, N. J., Ahn, K. H., Lee S. J., et al., Extension flow-induced red blood cell (RBC) deformation in the microchannel of contraction geometry. I. J. Vascular Biomedical Eng., 5, 2007, 8-13. C1005

[3] Dobbe, J. G. G., Hardemanb, M. R., Streekstrac, G. J., Strackeec, J., Inceb, C., and Grimbergen, C. A., Analyzing red blood cell-deformability distributions. Blood Cells, Molecules, and Diseases, 28, 2002, 373-284. doi:10.1006/bcmd.2002.0528 C1005

[4] Linderkamp, O., and Meiselman, H. J., Geometric, osmotic, and membrene mechanical properties of density-separated human red cells, Blood 59, 1982, 1121-1127. C1005

[5] Nash, G. B., and Wyard, S. J., Changes is surface area and volume measured by micropipette aspiration for erythrocytes ageing in vivo, Biorheology, 17, 1981, 479-484. C1005

[6] Corry, W. D., and Meiselman, H. J., Deformation of human erthrocytes in a centrifugal field, Biophysical Journal, 21, 1978, 19-34 C1005

[7] Eggleton, C. D., Popel, A. S., Large deformation of red blood cell ghosts in simple shear flow. Physics of Fluids, 10, 1998, 1823-1845. doi:10.1063/1.869703 C1006

[8] Abkarian, M. and Viallat, A., Vesicles and red blood cells in shear flow. Royal Society Chemistry, 4, 2008, 653-657. doi:10.1039/b716612e $\mathrm{C} 1006$ 
[9] Sheard, G. J., Leweke, T., Thompson, M. C., Hourigan, K., Flow around an impulsively arrested circular cylinder. Physics of Fluids, 19, 2007 article number 083601. doi:10.1063/1.2754346 C1010

[10] Sheard, G. J. \& Ryan, K., Pressure-driven flow past spheres moving in a circular tube. J. Fluid Mech., 592, 2007 233-262. doi:10.1017/S0022112007008543 C1010

[11] Sheard, G. J., Thompson, M. C. and Hourigan, K., From spheres to circular cylinders: the stability and flow structure of bluff ring wakes. J. Fluid Mech., 492, 2003, 147-180. doi:10.1017/S0022112003005512 C1010

[12] Press, W. H., Teukolsky, S. A. Vetterling, W. T., Flannery, B. P., Numerical Recipes in C: The Art of Scientific Computing, Second Edition. Cambridge University Press, 1988 (ISBN 0-521-43108-5) C1010

\section{Author addresses}

1. Simon P. Higgins, Division of Biological Engineering, Monash University, Victoria 3800, Australia.

2. Gregory J. Sheard, Department of Mechanical Engineering, Monash University, Victoria 3800, Australia.

3. Andreas Fouras, Division of Biological Engineering, Monash University, Victoria 3800, Australia. mailto:fouras@eng.monash.edu.au

4. Kerry Hourigan, Division of Biological Engineering, Monash University, Victoria 3800, Australia and Department of Mechanical Engineering, Monash University, Victoria 3800, Australia. 